\title{
Process of Interpretation of Two-Dimensional Densitometry Images for the Prediction of Bone Mechanical Strength
}

\author{
Laurent Pothuaud \\ INSERM, University Victor Segalen, Bordeaux, France \\ Pothuaudeaol.com
}

\section{Introduction}

The in vivo evaluation of Trabecular Bone Structure (TBS) is a major challenge in bone study. The modern definition of osteoporosis involves low Bone Mineral Density (BMD) and TBS alterations. BMD is well measured in clinical practice by DEXA (Double Energy X-ray Absorptiometry). On the other hand, TBS cannot be measured in routine at the present time. The aim of the present process is to exploit the increased quality of DEXA bone images in order to characterize the gray level properties of such bone projection images and to evaluate a parameter TBS by considerations of projection effects. Moreover, a mathematical model is proposed that allows to evaluate a combined parameter BDS (Bone Density Structure).

\section{In Vitro Validation}

In a preliminary in vitro study, it has been showed the potential of the new parameter BDS for the prediction of the ultimate constraint of bone $(\mathrm{Cu})$.

\section{Preliminary Results in Normal Postmenopausal Women}

The aim of this preliminary clinical study was to evaluate the changes in TBS and BDS parameters in two BMD matched groups with significant age difference. Two groups of 12 subjects each $\left(\mathrm{G}_{\mathrm{Y}}\right.$ and $\left.\mathrm{G}_{\mathrm{O}}\right)$ were constituted with mean age of 54.9+/-7.7 years $\left(G_{Y}\right)$ and $70.1+/-6.6$ years $\left(G_{O}\right)$, while neck-BMD was matched for the two groups: $0.647+/-0.072\left(\mathrm{G}_{\mathrm{Y}}\right)$ and $0.646+/-0.071\left(\mathrm{G}_{\mathrm{O}}\right)$. Significant differences were obtained between the two groups for both TBS and BDS parameters, with: TBS $=0.631+/-0.079 \quad\left(\mathrm{G}_{\mathrm{Y}}\right)$ versus TBS $=0.798+/-0.055 \quad\left(\mathrm{G}_{\mathrm{O}}\right), \quad \mathrm{p}<0.0001 \quad$ (t-test); $\mathrm{BDS}=0.222+/-0.022\left(\mathrm{G}_{\mathrm{Y}}\right)$ versus $\mathrm{BDS}=0.190+/-0.036\left(\mathrm{G}_{\mathrm{O}}\right), \mathrm{p}=0.015$ (t-test). These differences, in accordance with the exponential model, showed that with BMD constant an increase of TBS was associated with a decrease of BDS (aimed to predict the mechanical strength of bone). This result was in agreement with the fact that the older 
group $\left(\mathrm{G}_{\mathrm{O}}\right)$ would be less strong than the younger one $\left(\mathrm{G}_{\mathrm{Y}}\right)$, although their bone mineral densities (BMD) were identical.

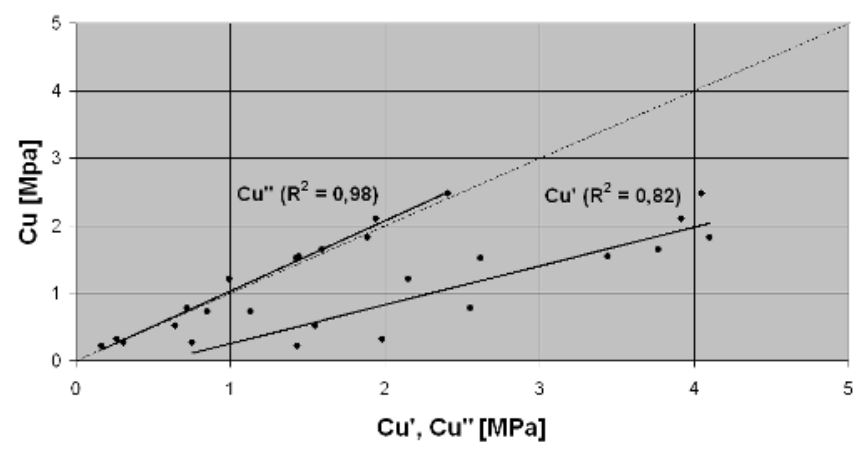

Fig. 1. The parameter BMD makes it possible to explain a relatively great part of the ultimate constraint $(\mathrm{Cu})$. The linear model $-\mathrm{Cu}^{\prime}=\mathrm{a}_{0}+\mathrm{a}_{1} * \mathrm{BMD}-$ allows to explain approximately $82 \%$ of the variations of $\mathrm{Cu}$ - experimental results obtained from a set of 13 trabecular bone samples. This relationship is however limited by the fact that various samples with very close densities can have different mechanical properties, just as control and osteoporotic subjects can have very close values of BMD. The exponential model $-\mathrm{Cu}=\mathrm{BDS}=\mathrm{b}_{0}+\mathrm{b}_{1} * \exp \left(\mathrm{b}_{2} * \mathrm{BMD}\right) * \mathrm{TBS}-$ makes it possible to increase the accuracy of the prediction of $\mathrm{Cu}$ explaining up to $98 \%$ of its variations.

\section{Discussion}

In this preliminary study, it has been firstly demonstrated the technical feasibility to evaluate a new parameter TBS directly from DEXA bone images. Furthermore, it has been showed the potential interest of the new proposed method of interpretation (BDS) to evaluate, more accurately than BMD alone, the quality of bone. This is a very new and original approach that does not necessitate supplementary exam for patient in benefiting from a well-standardized and well-reproducible imaging technique. This approach can be exploited in retrospective study as well. At present, only BMD is used to quantify the quality of bone. The prevention strategy then consists to compare the BMD value of a subject to two "age-matched" and "young normals" standards, giving a comparative indication on the remained amount of bone and on the kinetics of bone loss respectively. Nevertheless, it is well recognized that BMD alone is not sufficient to accurately predict the risk of fracture in individual subject. Further investigations, aimed to establish normative references of TBS and BDS in larger normal women population are in progress. Such normative references would permit to define more reliable risk factor to increase the prevention strategy of osteoporotic fractures. 